PROCEEDINGS OF THE

AMERICAN MATHEMATICAL SOCIETY

Volume 128, Number 3, Pages 853-855

$\mathrm{S}$ 0002-9939(99)05222-3

Article electronically published on July 28, 1999

\title{
A NOTE ON THE \\ CONTACT ANGLE BOUNDARY CONDITION FOR MONGE-AMPÈRE EQUATIONS
}

\author{
JOHN URBAS
}

(Communicated by Peter Li)

\begin{abstract}
We give a simple proof of a result of Xinan Ma concerning a necessary condition for the solvability of the two-dimensional Monge-Ampère equation subject to the contact angle or capillarity boundary condition. Our technique works for more general Monge-Ampère equations in any dimension, and also extends to some other boundary conditions.
\end{abstract}

In [1] Xinan Ma considered the Monge-Ampère equation

$$
\operatorname{det} D^{2} u=c \text { in } \Omega \text {, }
$$

where $\Omega$ is a $C^{2}$ bounded convex domain in $\mathbf{R}^{2}$ and $c$ is a positive constant, subject to the contact angle or capillarity boundary condition

$$
D_{\nu} u=\cos \theta \sqrt{1+|D u|^{2}} \quad \text { on } \quad \partial \Omega,
$$

where $\nu$ denotes the outer unit normal vectorfield to $\partial \Omega$ and $\theta \in(0, \pi / 2)$ is a constant. The result of [1] is that if there is a convex solution $u \in C^{3}(\Omega) \cap C^{2}(\bar{\Omega})$ of (1), (2), then

$$
k_{0} \leq \max \{\sqrt{c} \cos \theta, \sqrt{c} \tan \theta\},
$$

where $k_{0}$ denotes the minimum curvature of $\partial \Omega$.

Here we provide a much simpler and more transparent proof of this result, which moreover, generalizes to more general Monge-Ampère equations in higher dimensions and to some other boundary conditions.

Theorem. Let $\Omega$ be a bounded domain in $\mathbf{R}^{n}$ with $C^{2}$ boundary, $f \in C(\bar{\Omega})$ a positive function and $\phi \in C(\partial \Omega)$ with $0<\phi<1$. If $u \in C^{2}(\Omega) \cap C^{1}(\bar{\Omega})$ is a convex solution of

$$
\begin{gathered}
\operatorname{det} D^{2} u=f \quad \text { in } \quad \Omega, \\
D_{\nu} u=\phi \sqrt{1+|D u|^{2}} \quad \text { on } \quad \partial \Omega,
\end{gathered}
$$

Received by the editors May 12, 1998.

1991 Mathematics Subject Classification. Primary 35J25, 35J60, 35J65.

Key words and phrases. Monge-Ampère equations, contact angle boundary condition.

(C)1999 American Mathematical Society 
then

$$
k_{0} \leq \frac{\left(\sup _{\Omega} f\right)^{1 / n}}{\inf _{\partial \Omega} \frac{\phi}{\sqrt{1-\phi^{2}}}}
$$

where $k_{0}$ denotes the minimum normal curvature of $\partial \Omega$.

Remark. If $f=c$ and $\phi=\cos \theta$ where $c>0$ and $\theta \in(0, \pi / 2)$ are constants, (6) reduces to

$$
k_{0} \leq c^{1 / n} \tan \theta,
$$

which improves (3) for small enough $\theta$. In addition, it will be clear from the proof below that equality holds in (7) if $\Omega$ is a ball.

Proof of Theorem. We may assume that $k_{0}>0$; otherwise the result is immediate. By the definition of $k_{0}$ it is clear that $\Omega$ is contained in a ball of radius $k_{0}^{-1}$. Next we observe that since $\phi>0$, we have $D_{\nu} u>0$ on $\partial \Omega$, so $u$ must have its minimum at an interior point of $\Omega$, say at $x_{0} \in \Omega$. Thus $D u\left(x_{0}\right)=0$. Now, by integrating (4) and using the classical change of variables formula we obtain

$$
\begin{aligned}
|D u(\Omega)| & =\int_{\Omega} \operatorname{det} D^{2} u \\
& \leq|\Omega| \sup _{\Omega} f \\
& \leq \omega_{n} k_{0}^{-n} \sup _{\Omega} f,
\end{aligned}
$$

where $\omega_{n}$ denotes the volume of the unit ball in $\mathbf{R}^{n}$. Since $D u(\Omega)$ is an open set containing the origin, there is a point $y_{0} \in \partial \Omega$ such that

$$
\left|D u\left(y_{0}\right)\right| \leq k_{0}^{-1}\left(\sup _{\Omega} f\right)^{1 / n} .
$$

On the other hand, from the boundary condition (5) we obtain

$$
\left|D u\left(y_{0}\right)\right| \geq \inf _{\partial \Omega} \frac{\phi}{\sqrt{1-\phi^{2}}} .
$$

(9) and (10) clearly imply the estimate (6).

Remarks. (i) A similar argument can be used if $u \in C^{2}(\Omega) \cap C^{1}(\bar{\Omega})$ is a convex solution of

$$
\operatorname{det} D^{2} u \leq f(x) / g(D u) \quad \text { in } \quad \Omega
$$

for some positive functions $f \in L^{p}(\Omega), p>1$, and $g \in L_{l o c}^{1}\left(\mathbf{R}^{n}\right)$ with $\int_{\mathbf{R}^{n}} g=\infty$. Similar to before we have

$$
\begin{aligned}
\int_{D u(\Omega)} g & \leq \int_{\Omega} f \\
& \leq|\Omega|^{1-1 / p}\|f\|_{p} \\
& \leq\left(\omega_{n} k_{0}^{-n}\right)^{1-1 / p}\|f\|_{p} .
\end{aligned}
$$

We may now choose $R>0$ so large that

$$
G(R):=\int_{B_{R}(0)} g=\left(\omega_{n} k_{0}^{-n}\right)^{1-1 / p}\|f\|_{p} .
$$


As before, since $D u(\Omega)$ is an open set containing the origin, there is some $y_{0} \in$ $\partial \Omega$ such that $\left|D u\left(y_{0}\right)\right| \leq R$. Combining this with (10) we obtain, after some rearrangement,

$$
k_{0} \leq \frac{\omega_{n}^{1 / n}\|f\|_{p}^{p / n(p-1)}}{\left(G\left(\inf _{\partial \Omega} \frac{\phi}{\sqrt{1-\phi^{2}}}\right)\right)^{p / n(p-1)}} .
$$

This reduces to (6) in the special case that $f \in L^{\infty}(\Omega), g \equiv 1$, if we let $p \rightarrow \infty$.

(ii) The same argument can be applied to the linear boundary condition

$$
D_{\beta} u=\phi \quad \text { on } \quad \partial \Omega
$$

where $\beta$ is a strictly oblique unit vector field on $\partial \Omega$ and $\phi>0$; the quantity $\inf _{\partial \Omega} \frac{\phi}{\sqrt{1-\phi^{2}}}$ need only be replaced by $\inf _{\partial \Omega} \phi$ in (6) and (11). Clearly, (5) could also be replaced by a variety of other boundary conditions having an appropriate structure.

\section{REFERENCES}

[1] Xinan Ma, A necessary condition of solvability for the capillarity boundary of Monge-Ampère equations in two dimensions, Proc. Amer. Math. Soc. (to appear). CMP 98:05

Centre for Mathematics and its Applications, School of Mathematical Sciences, Australian National University, Canberra ACT 0200, Australia

E-mail address: John.Urbas@maths.anu.edu.au 